\title{
Elastic pp scattering from the optical point to past the dip: an empirical parametrization from ISR to LHC
}

\author{
D. A. Fagundest \\ INFN Frascati National Laboratories, Via E. Fermi 40, 00444, Italy and \\ Instituto de Física Gleb Wataghin, Universidade Estadual de Campinas, UNICAMP, 13083-859 Campinas SP, Brazil \\ G. Pancher \\ INFN Frascati National Laboratories, Via E. Fermi 40, 00444, Italy
}

A. Grau节

Departamento de Física Teórica y del Cosmos, Universidad de Granada, 18071 Granada, Spain

S. Pacett $\$$ and Y. N. Srivastava

Physics Department and INFN, University of Perugia, 06123 Perugia, Italy

\begin{abstract}
We describe the main features of recent LHC data on elastic $p p$ scattering through a simple parametrization to the amplitude, inspired by a model proposed by Barger and Phillips in 1973, comprising of two exponentials with a relative phase. Despite its simplicity, this parameterization reproduces two essential aspects of the elastic differential cross section, the well known precipitous descent in the forward direction and a sharp 'dip' structure. To include a complete description of data sets near $-t=0$, we correct the original parametrization. We examine two possibilities, the presence of the two-pion threshold singularity or a multiplicative factor reflecting the proton form factor. We find good descriptions of LHC7 and ISR data in either case. The form factor model allows simple predictions for higher energies through asymptotic theorems and asymptotic sum rules in impact parameter space. We present predictions for this model at higher LHC energies, which can be used to test whether asymptotia is reached. The black disk limit in this model is seen to be reached only for $\sqrt{s} \sim 10^{6} \mathrm{TeV}$.
\end{abstract}

PACS numbers: 13.75.Cs, 13.85.-t

Keywords: Elastic cross section, Asymptotia

\begin{tabular}{rl|}
$*$ & fagundes@ifi.unicamp.br \\
$\dagger$ & giulia.pancheri@lnf.infn.it \\
$\ddagger$ & igrau@ugr.es \\
$\S$ & simone.pacetti@pg.infn.it \\
$\uparrow$ & yogendra.srivastava@pg.infn.it
\end{tabular} 


\section{CONTENTS}

I. Introduction

II. The Barger and Phillips model and LHC7 data

III. The proton form factor modification: $m B P 2$

III.1. Slope parameter in the modified models

IV. Asymptotic predictions of the empirical model $m B P 2$

Acknowledgments

A. Two-pion threshold effects on the BP model: $m B P 1$

B. Other form factor modifications of the Barger and Phillips model

\section{INTRODUCTION}

The total $p p$ cross-section and the elastic differential cross-section offer a unique opportunity to study confinement and the transition to perturbative QCD, as they are influenced by large and small distances.

We now have data for the total and the elastic differential cross section from LHC running at $\sqrt{s}=7 \mathrm{TeV}(\mathrm{LHC} 7)$ [1. Data from LHC running at $\sqrt{s}=8 \mathrm{TeV}$ (LHC8), soon to be available, and $14 \mathrm{TeV}$ (LHC14) may be our last chance to explain $p p$ scattering in fundamental terms. A tool to help in this endeavor is a good phenomenological understanding of their energy behavior, without the bias imposed by models. To present one such phenomenological description is the aim of this paper.

In what follows we shall propose an empirical description of the differential elastic $p p$ cross-section to be used at LCH8 and LHC14. This description follows from the original proposal by Barger and Phillips (BP) [2], who described ISR data with a 5 parameters fit, i.e. writing the scattering amplitude as

$$
\mathcal{A}(s, t)=i\left[\sqrt{A(s)} e^{B(s) t / 2}+e^{i \phi(s)} \sqrt{C(s)} e^{D(s) t / 2}\right]
$$

In [3], we had applied this parametrization to preliminary TOTEM results at LHC7 elastic differential cross-section data 4]. In this paper we refine that analysis presenting an improved description of published data 1], which includes the very small $-t$ value, i.e. parametrizing both the total and the elastic cross-section within a few percent of the present LHC7 data. We also propose its extension to higher energies, providing a parametrization obeying asymptotic theorems [5, 6] and apply it to study the black disk limit approach.

\section{THE BARGER AND PHILLIPS MODEL AND LHC7 DATA}

The parametrization, given in Eq. (1), corresponds to a complex amplitude, which is composed of two terms, and a relative phase $\phi$, which was found phenomenologically to be $\sim 2.8 \mathrm{rad}$ at LHC7, and can be interpreted as corresponding to contributions from opposite parities, $C= \pm 1$. The publication of the actual data by the part of the TOTEM collaboration [1] requires an update and also a revision of the analysis we performed to preliminary TOTEM data in [3],7]. Applying Eq. (1) to the published data and using the same parameters of [3], we find that, when 
both statistical and systematic errors are included in the fit, the description is still acceptable with $\chi^{2} / D O F \sim 2.6$. However, when the analysis is performed with only statistical errors, the $\chi^{2}$ for the entire range becomes unacceptably large. In particular, the parameterization of Eq.(1) reproduces poorly the measured value of the total cross-section at LHC7. The problem therefore seems to lie with the optical point. To pinpoint the origin of the problem, we have fitted the now released data [1] implementing different cuts of $t_{\min }$ for which the BP model provides a suitable description. Specifically, any result with $\chi^{2} / D O F \lesssim 3$ is considered acceptable. In Table I we display a grid of possible cuts and the respective $\chi^{2} / D O F$ values. We also calculate the corresponding values obtained for the differential cross-section at the optical point and the total cross-section. We find that, when the fit with the BP amplitude is able to reproduce the optical point, the statistical description is not very good. On the other hand, the fit becomes quite good for 0.2 $\mathrm{GeV}^{2}<\left|t_{\text {min }}\right|<0.3 \mathrm{GeV}^{2}$, even though the total cross-section obtained in these cases is too low.

TABLE I. Statistical results of fits with simple BP model of Eq. 11, with $\chi^{2}$ calculated for the range $-t>-t_{m i n}$ and resulting values for the optical point and the total cross section.

\begin{tabular}{c|c|c||c|c}
\hline \hline$-t_{\min }\left(\mathrm{GeV}^{2}\right)$ & $D O F$ & $\chi^{2} / D O F$ & $d \sigma_{e l} /\left.d t\right|_{t=0}\left(\mathrm{mbGeV}^{-2}\right)$ & $\sigma_{\text {tot }}(\mathrm{mb})$ \\
\hline 0.01 & 156 & 9.40 & 490.2 & 97.9 \\
\hline 0.10 & 118 & 6.33 & 422.8 & 90.9 \\
\hline 0.20 & 94 & 2.66 & 282.0 & 74.2 \\
\hline 0.30 & 80 & 1.62 & 181.8 & 59.6 \\
\hline 0.40 & 70 & 1.41 & 212.1 & 64.4 \\
\hline \hline
\end{tabular}

From Table $\mathrm{I}$ we conclude that, past the very small $-t<0.2 \mathrm{GeV}^{2}$ values, the parametrization of Eq. (1) is suitable to describe two essential features of the differential elastic cross section at high energies, namely the dip structure and the larger $|t|$ region, as one can also see from Fig. 1. Notice that the exponential fit in the range $|t|>1.0 \mathrm{GeV}^{2}$ can be taken to be as good as the power law fit $|t|^{-n}$ presented by the TOTEM Collaboration in [4], as shown in the inset of Fig. 1.

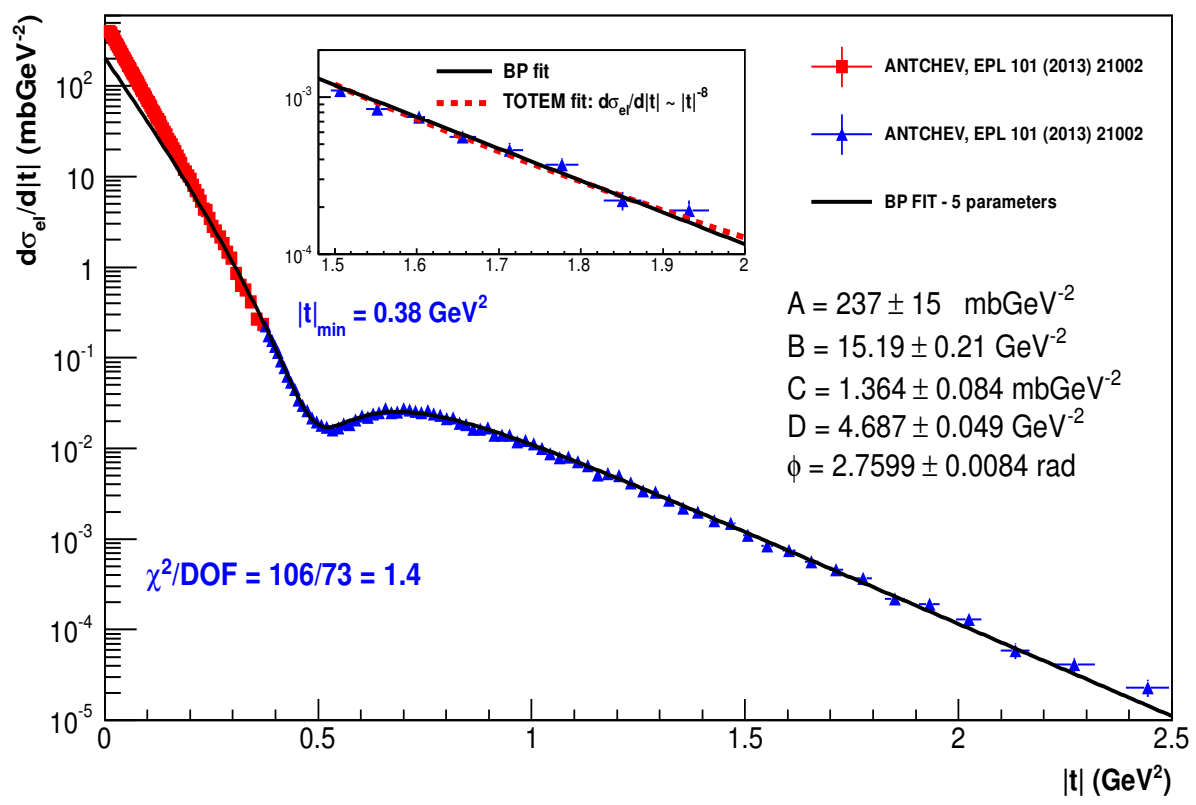

FIG. 1. Fit to the differential $p p$ cross-section at $7.0 \mathrm{TeV}$ [1] with the BP parametrization of Eq. (1) in the range $0.38 \leq|t| \leq 2.4$ $\mathrm{GeV}^{2}$ with $\chi^{2} /$ d.o.f in this interval. Inset: the power law fit $|t|^{-n}$, with $n \approx 8$, compared to the exponential fit in the range $1.5 \leq|t| \leq 2.0 \mathrm{GeV}^{2}$.

We notice at this point that, for the BP model of Eq. (1) to give a good global description from the optical point to past the dip, the very small $|t|$ behavior must receive a correction, while, at the same time, the region past $-t=0.2 \mathrm{GeV}^{2}$ should still be described through two exponentials (and the phase). Namely, since the BP model 
gives a very good description of LHC7 data except that in the forward region, there is no phenomenological reason to modify it neither around the dip nor in the tail. We thus propose to ameliorate the very small $-t$ behavior by modifying only the first term in Eq. (1) with a factor $G(s, t)$ such that $G(s, 0)=1$, and suggest to parametrize existing and future $p p$ data with the amplitude:

$$
\mathcal{A}(s, t)=i\left[G(s, t) \sqrt{A(s)} e^{B(s) t / 2}+e^{i \phi(s)} \sqrt{C(s)} e^{D(s) t / 2}\right] .
$$

We have examined two possibilities:

- a factor $G(s, t)=\exp \left[-\gamma(s)\left(\sqrt{4 \mu^{2}-t}-2 \mu\right)\right]$ reflecting the presence of the nearest $t$-channel singularity, i.e. the two pion threshold [8, 9] discussed in [10] and [11, labeling this possibility as the $m B P 1$ model,

- a factor $G(s, t)=F_{P}^{2}=1 /\left(1-t / t_{0}\right)^{4}$ modeled after the proton form factor, which describes phenomenologically the probability that the proton breaks up as the squared momentum transfer increases, labeling this as $m B P 2$ model.

The $m B P 1$ model, and other possible modifications of the BP models are discussed in detail in Appendices $\mathrm{A}$ and $\mathrm{B}$ of this paper. In the appendices we shall also present further details, such as analytic expressions for the elastic cross-section and, in Appendix $[$ ] the impact parameter profile functions for both $m B P 1$ and $m B P 2$ models.

\section{THE PROTON FORM FACTOR MODIFICATION: $M B P 2$}

In this section, and the ones to follow, we present here a modification of the BP model at very small $t$-values obtained through the proton form factor. As viable parametrization of LHC data, we analyze the physics content of the following model for the elastic scattering amplitude:

$$
\mathcal{A}(s, t)=i\left[F_{P}^{2}(t) \sqrt{A(s)} e^{B(s) t / 2}+e^{i \phi(s)} \sqrt{C(s)} e^{D(s) t / 2}\right]
$$

with $A, B, C, D, \phi$ and $t_{0}$ as free parameters. We display our results with this model (henceforth called $m B P 2$ ) in Fig. 2. ISR data sets used in the fits comprise the data collection by Amaldi and Schubert [12] and all experimental information available from 1980 onwards [13 16]. LHC7 data are from [1].
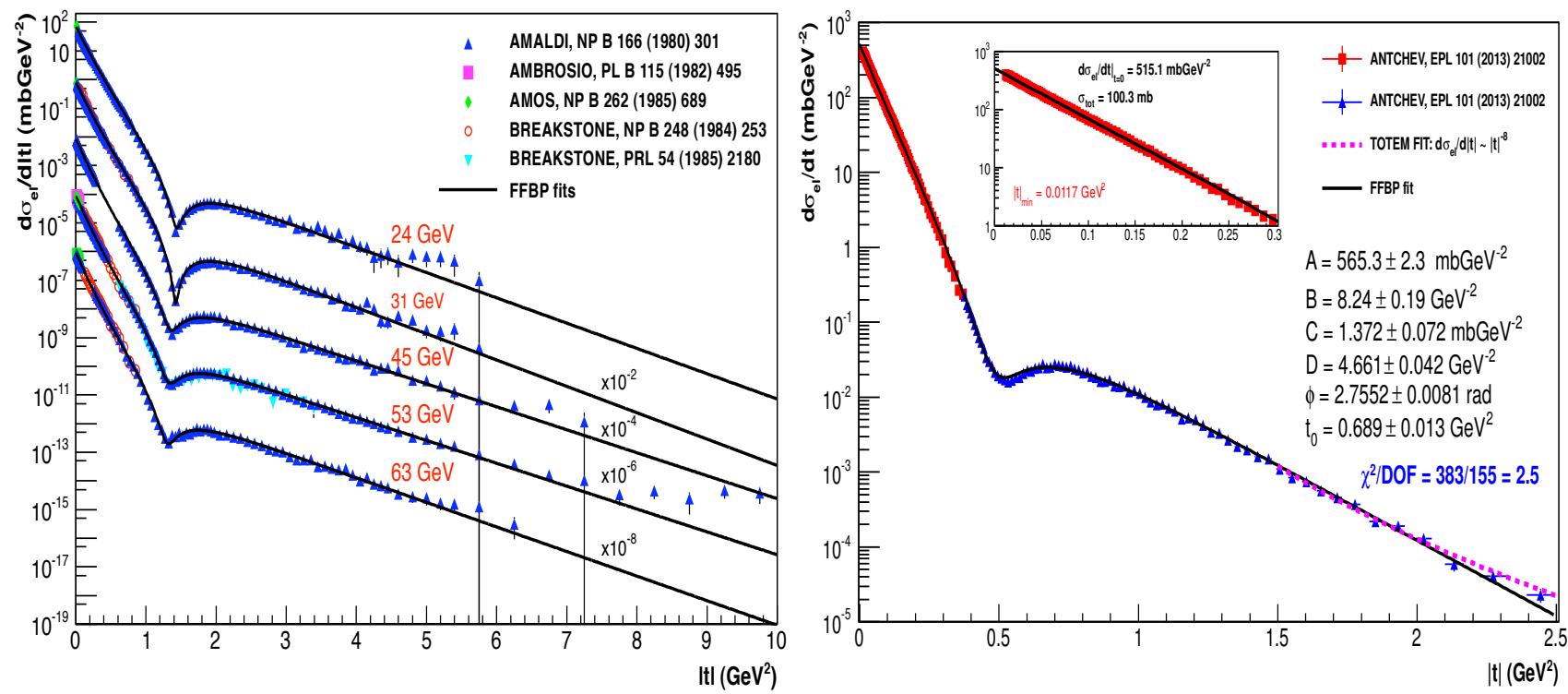

FIG. 2. Fits to the ISR and LHC7 data sets with model $m B P 2$, and $t_{0}$ a free parameter. Data sets as described in the text.

We summarize the results of the fit in Table II] with the last two rows corresponding to the fit to LHC7 data obtained by using for $t_{0}$ the results from the fit or keeping $t_{0}$ fixed at $0.71 \mathrm{GeV}^{2}$. Plots and fits results for the model with the two pion threshold correcting the $t \sim 0$ behavior can be found in Appendix A. 
TABLE II. First six rows give values of free fit parameters $A, B, C, D, t_{0}$ and $\phi$ for the model $m B P 2$ at each energy analyzed. In the last row, the scale parameter $t_{0}$ is kept fixed. $A$ and $C$ are expressed in units $\mathrm{mbGeV}^{-2}, B$ and $D$ in units $\mathrm{GeV}^{-2}, t_{0}$ in units $\mathrm{GeV}^{2}$ and $\phi$, in radians.

\begin{tabular}{|c|c|c|c|c|c|c||c|c|}
\hline \hline$\sqrt{s}(\mathrm{GeV})$ & $A$ & $B$ & $C\left(\times 10^{-3}\right)$ & $D$ & $t_{0}$ & $\phi$ & $D O F$ & $\frac{\chi^{2}}{\mathrm{DOF}}$ \\
\hline 24 & $74.8 \pm 0.8$ & $4.0 \pm 0.1$ & $4.8 \pm 0.7$ & $2.03 \pm 0.06$ & $1.06 \pm 0.03$ & $3.31 \pm 0.01$ & 128 & 1.2 \\
\hline 31 & $83.7 \pm 0.2$ & $3.90 \pm 0.07$ & $5.4 \pm 0.5$ & $2.12 \pm 0.04$ & $0.99 \pm 0.01$ & $3.06 \pm 0.01$ & 200 & 1.6 \\
\hline 45 & $89.6 \pm 0.2$ & $4.27 \pm 0.05$ & $2.4 \pm 0.2$ & $1.84 \pm 0.02$ & $0.912 \pm 0.009$ & $2.83 \pm 0.01$ & 201 & 3.7 \\
\hline 53 & $93.0 \pm 0.1$ & $4.51 \pm 0.05$ & $2.5 \pm 0.1$ & $1.84 \pm 0.01$ & $0.947 \pm 0.008$ & $2.79 \pm 0.01$ & 313 & 4.7 \\
\hline 63 & $97.4 \pm 0.2$ & $4.3 \pm 0.1$ & $3.5 \pm 0.4$ & $1.97 \pm 0.04$ & $0.90 \pm 0.01$ & $2.86 \pm 0.06$ & 159 & 2.1 \\
\hline 7000 & $565 \pm 2$ & $8.2 \pm 0.2$ & $1370 \pm 70$ & $4.66 \pm 0.04$ & $0.69 \pm 0.01$ & $2.755 \pm 0.008$ & 155 & 2.5 \\
\hline 7000 & $562 \pm 1$ & $8.54 \pm 0.03$ & $1280 \pm 34$ & $4.61 \pm 0.03$ & 0.71 (fixed) & $2.744 \pm 0.004$ & 156 & 2.5 \\
\hline \hline
\end{tabular}

We notice that the value of the parameter $t_{0}$ is larger at ISR energies than at LHC7, where its value is consistent with $F_{P}(t)$ being the EM form factor, i.e. $t_{0} \sim 0.71 \mathrm{GeV}^{2}$. In fact, fits to the LHC7 data with this value give a $\chi^{2} / \mathrm{DOF}=2.5$ just as in the case of the free fit. This difference between ISR and LHC probably corresponds to low energy contributions to this parameter. We make the ansatz that when asymptotic energies are reached, $t_{0}$ correspond to its EM form factor value. At non-asymptotic energies, the parameter $t_{0}$ can be parametrized as shown in Fig. 3 . In this figure, elsewherelse in this paper and unless otherwise stated, the squared energies $s$ in $\ln s$ are in units of $G e V^{2}$.

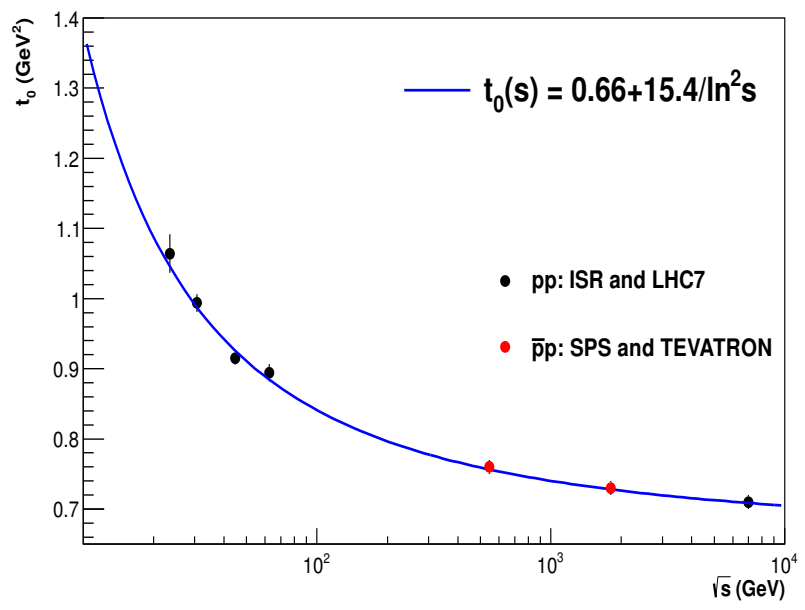

FIG. 3. Fit to the energy dependence of the scale parameter $t_{0}$. The black points correspond to results from Table II for $p p$. The red dots indicate the value taken by this parameterization for $t_{0}$ at energies corresponding to $S \bar{p} p$ and the TeVatron, where the process is $\bar{p} p$.

From the $m B P 2$ model the analytical expression for the elastic cross section follows:

$$
\sigma_{e l}(s)=A t_{0} e^{B t_{0}} E_{8}\left(B t_{0}\right)+\frac{C}{D}+2(\sqrt{A C} \cos \phi) t_{0} e^{(B+D) t_{0} / 2} E_{4}\left(\frac{(B+D) t_{0}}{2}\right)
$$

with $E_{n}(x)=\int_{1}^{\infty} d y e^{-x y} / y^{n}$. In Table III we present the values of the total and elastic cross sections as obtained from both models, $m B P 1$ and $m B P 2$, together with the optical point for LHC7 and ISR energies. Parameter values for the $m B P 1$ model can be found in Appendix A 
TABLE III. Cross sections and the optical point following from models $m B P 1$ and $m B P 2$.

\begin{tabular}{c|c|c|c|c}
\hline \hline Model & $\sqrt{s}(\mathrm{GeV})$ & $\sigma_{\text {tot }}(\mathrm{mb})$ & $\sigma_{e l}(\mathrm{mb})$ & $d \sigma_{e l} /\left.d t\right|_{t=0}\left(\mathrm{mbGeV}^{-2}\right)$ \\
\hline & 24 & 40.0 & 6.80 & 82.0 \\
& 31 & 40.6 & 7.15 & 84.3 \\
& 45 & 42.1 & 7.14 & 90.9 \\
& 53 & 42.9 & 7.43 & 94.0 \\
& 63 & 43.7 & 7.68 & 97.8 \\
& 7000 & 101 & 25.6 & 524 \\
\hline \multirow{5}{*}{ BP2 } & 24 & 37.9 & 6.65 & 73.6 \\
& 31 & 40.1 & 7.20 & 82.4 \\
& 45 & 41.6 & 7.13 & 88.7 \\
& 53 & 42.4 & 7.42 & 92.1 \\
& 63 & 43.3 & 7.60 & 96.3 \\
\hline \hline
\end{tabular}

We shall now apply to the modified amplitude the asymptotic sum rules presented in our previous analysis 3 . The sum rules correspond to the ansatz of total absorption in $b$-space. Namely, $S R_{1} \equiv \Im m \tilde{\mathcal{A}}(s, b=0)=1$ and $S R_{0} \equiv \Re e \tilde{\mathcal{A}}(s, b=0)=0$ at asymptotic energies, where $\tilde{\mathcal{A}}(b, s)$ is the Fourier transform of the scattering amplitude in $b$-space. For the $m B P 1$ model, the analytical expressions for the sum rules for imaginary and real part of the amplitude are presented in Appendix A. As discussed in [3, for the satisfaction of the first sum rule, $S R_{0}=0$, it is necessary to introduce a real part for the first term, the one dominant at small $-t$, for which $C=+1$. Let us denote with $\hat{\rho}(s)$ the contribution to the ratio of the real to the imaginary part of the first term. For $m B P 2$ the sum rules give the following results

$$
\begin{array}{r}
S R_{1}=\frac{1}{2 \sqrt{\pi}} \int_{o}^{\infty} d T\left[\sqrt{\frac{A}{1+\hat{\rho}^{2}}} \frac{e^{-B T / 2}}{\left[1+\left(T / t_{o}\right)\right]^{4}}-\sqrt{C} e^{-D T / 2}|\cos \phi|\right]= \\
=\frac{1}{\sqrt{\pi}}\left[-\frac{\sqrt{C}}{D}|\cos \phi|+\sqrt{\frac{A}{1+\hat{\rho}^{2}}} \frac{t_{0}}{2} e^{B t_{0} / 2} E_{4}\left(B t_{0} / 2\right)\right] \\
S R_{0}=\frac{1}{\sqrt{\pi}}\left[-\frac{\sqrt{C}}{D} \sin \phi+\sqrt{\frac{A}{1+\hat{\rho}^{2}}} \hat{\rho} \frac{t_{0}}{2} e^{B t_{0} / 2} E_{4}\left(B t_{0} / 2\right)\right]
\end{array}
$$

Using the tight bound

$$
\frac{1}{x+n}<\left[e^{x} E_{n}(x)\right]<\frac{1}{x+n-1} ; n=1,2, \ldots
$$

we have

$$
\left[\frac{1}{B+8 / t_{0}}\right]<\frac{t_{0}}{2} e^{B t_{0} / 2} E_{4}\left(B t_{0} / 2\right)<\left[\frac{1}{B+6 / t_{0}}\right] .
$$

Hence, a simple analytical result for the sum rules can be obtained in the modified $m B P 2$ model by taking the mean value 7 in the denominator, so that

$$
\begin{aligned}
& S R_{1}=\frac{1}{\sqrt{\pi}}\left[-\frac{\sqrt{C}}{D}|\cos \phi|+\frac{\sqrt{\frac{A}{1+\hat{\rho}^{2}}}}{\hat{B}}\right] ; \hat{B}=B+\frac{7}{t_{0}} \\
& S R_{0}=\frac{1}{\sqrt{\pi}}\left[-\frac{\sqrt{C}}{D} \sin \phi+\frac{\sqrt{\frac{A}{1+\hat{\rho}^{2}}}}{\hat{B}} \hat{\rho}\right] ; \hat{B}=B+\frac{7}{t_{0}} .
\end{aligned}
$$

Asymptotically, we expect the following:

$$
S R_{1} \rightarrow 1-; S R_{0} \rightarrow 0+
$$


In order to estimate the values taken by $S R_{0}$ and $S R_{1}$ and check whether total absorption has been taking place, an estimate for $\hat{\rho}$ is needed. To this aim we use the soft $k_{t}$ resummation model of Ref. [17] where the leading term of the cross-section is driven by QCD mini-jets. In this model the asymptotic behavior of the total cross-section is obtained as $\sigma_{\text {total }} \sim(\ln s)^{1 / p}$ [18, where the parameter $p$ controls the large $b$ - behavior of the impact parameter distribution and obeys the constraint $1 / 2<p<1$. Asymptotically then, $\hat{\rho}(s)=\pi / 2 p \ln s$.

We show in Table IV] the numerical results for $S R_{1}$ and $S R_{0}$ for both models, $m B P 1$, where the small $-t$ modification is obtained through a term reflecting the two pion loop singularity, and $m B P 2$, where the form factor is dominating the $t \simeq 0$ behavior.

TABLE IV. Sum rules for modified BP models at ISR23, ISR53 and LHC7.

\begin{tabular}{c|c|c|c|c}
\hline \hline Model & $p$ & $\sqrt{s}(\mathrm{GeV})$ & $S R_{1}$ & $S R_{0}$ \\
\hline & - & 24 & 0.721 & 0.011 \\
$m B P 1$ & - & 53 & 0.722 & 0.049 \\
& 0.66 & 7000 & 0.953 & 0.067 \\
& 0.77 & 7000 & 0.956 & 0.046 \\
\hline & - & 24 & 0.719 & 0.021 \\
$m B P 2$ & - & 53 & 0.717 & 0.049 \\
& 0.66 & 7000 & 0.950 & 0.070 \\
& 0.77 & 7000 & 0.953 & 0.048 \\
\hline \hline
\end{tabular}

The table indicates that the modified models ameliorate the satisfaction of the Sum Rules with respect to the simpler BP parametrization, but the asymptotic value $S R_{1}=1$ is not yet reached.

We also notice that in the BP model (and in its modified versions as well), the parameter $\rho(s)$, real to imaginary part in the forward direction, is given by

$$
\rho(s)=\frac{\hat{\rho}-\sqrt{\left(\frac{C}{A}\right)} \sin \phi}{1-\sqrt{\left(\frac{C}{A}\right)}|\cos \phi|} \longrightarrow \hat{\rho}+\sqrt{\left(\frac{C}{A}\right)}[\hat{\rho}|\cos \phi|-\sin \phi] .
$$

for $\sqrt{C / A}<<1$. We shall make use of Eq. $\sqrt{13}$ when discussing asymptotic predictions.

Before leaving this section, we present the results one obtains when the model $m B P 2$ is applied to elastic $\bar{p} p$ data. Following the previous comments, the scale $t_{0}$ has been fixed from the parametrization shown in Fig. 3 . We show the results of the fits to $\bar{p} p$ data in Fig. 4 .
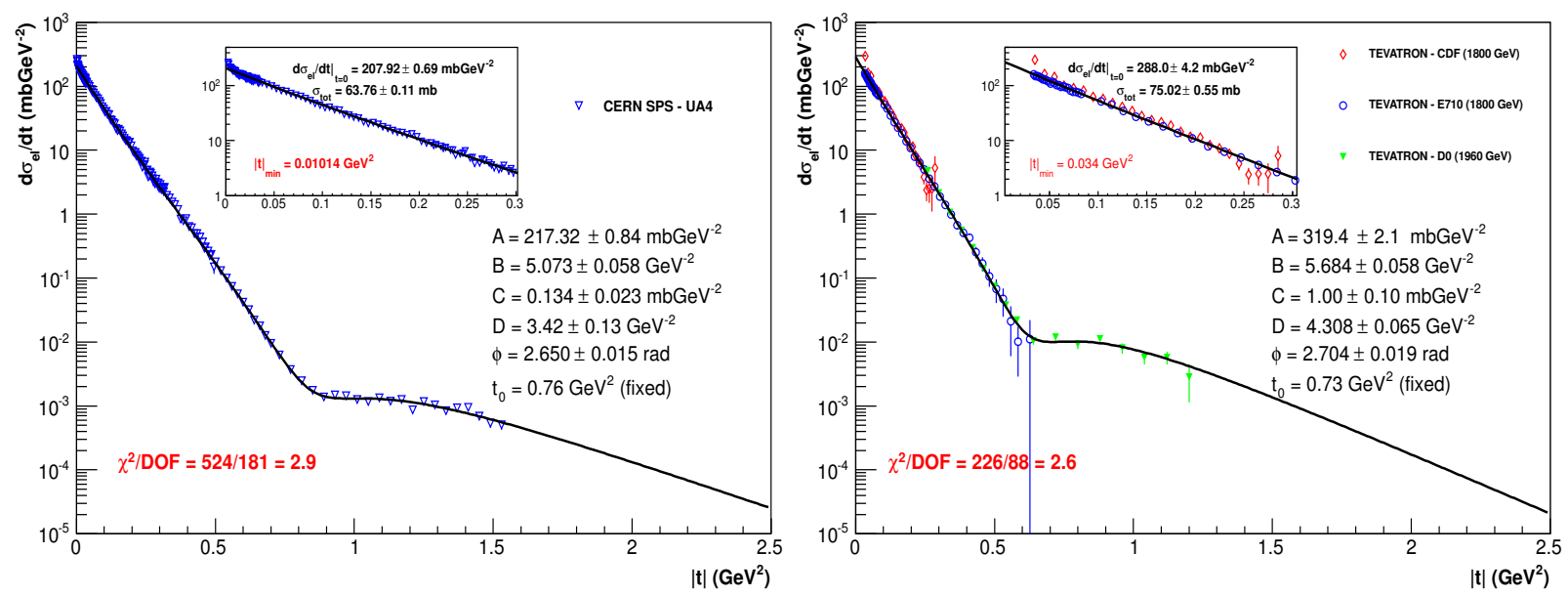

FIG. 4. The $m B P 2$ model applied to $\bar{p} p$ data. Value for parameters thus obtained are shown in the plots.

We note the absence of a distinctive dip in $\bar{p} p$, but also its faint appearance as the energy increases. 


\section{III.1. Slope parameter in the modified models}

The introduction of a general factor, $G(s, t)$, given either as in Eq. A2 or by the square of the proton form factor, leads to a change of curvature in the local slope. This behavior should be expected since the new model is influenced by $G(s, t)$ as follows:

$$
\begin{aligned}
B_{e f f}(s, t) & =\left(\frac{d \sigma_{e l}}{d t}\right)^{-1}\left[A B e^{B t} G^{2}(s, t)+2 A e^{B t} G(s, t) \frac{d G(s, t)}{d t}+C D e^{D t}\right. \\
& \left.+\sqrt{A C}(B+D) G(s, t) e^{(B+D) t / 2} \cos \phi+2 \sqrt{A C} e^{(B+D) t / 2} \frac{d G(s, t)}{d t} \cos \phi\right] .
\end{aligned}
$$

In Fig. 5 we display data for the effective forward slope $B_{\text {eff }}(s) \equiv B_{\text {eff }}(s, t=0)$, compared with the local slope $B_{e f f}(s, t)$, at ISR53 and LHC7, following from the above-mentioned models. We notice from this figure that the modification with the square root in the exponential, $m B P 1$, appears to overrate near-forward slopes. In fact, the respective forward slopes exceed by some $10 \%$ the measurements at ISR 53 and LHC7. This provides yet another reason to focus on the form factor modified model, $m B P 2$.
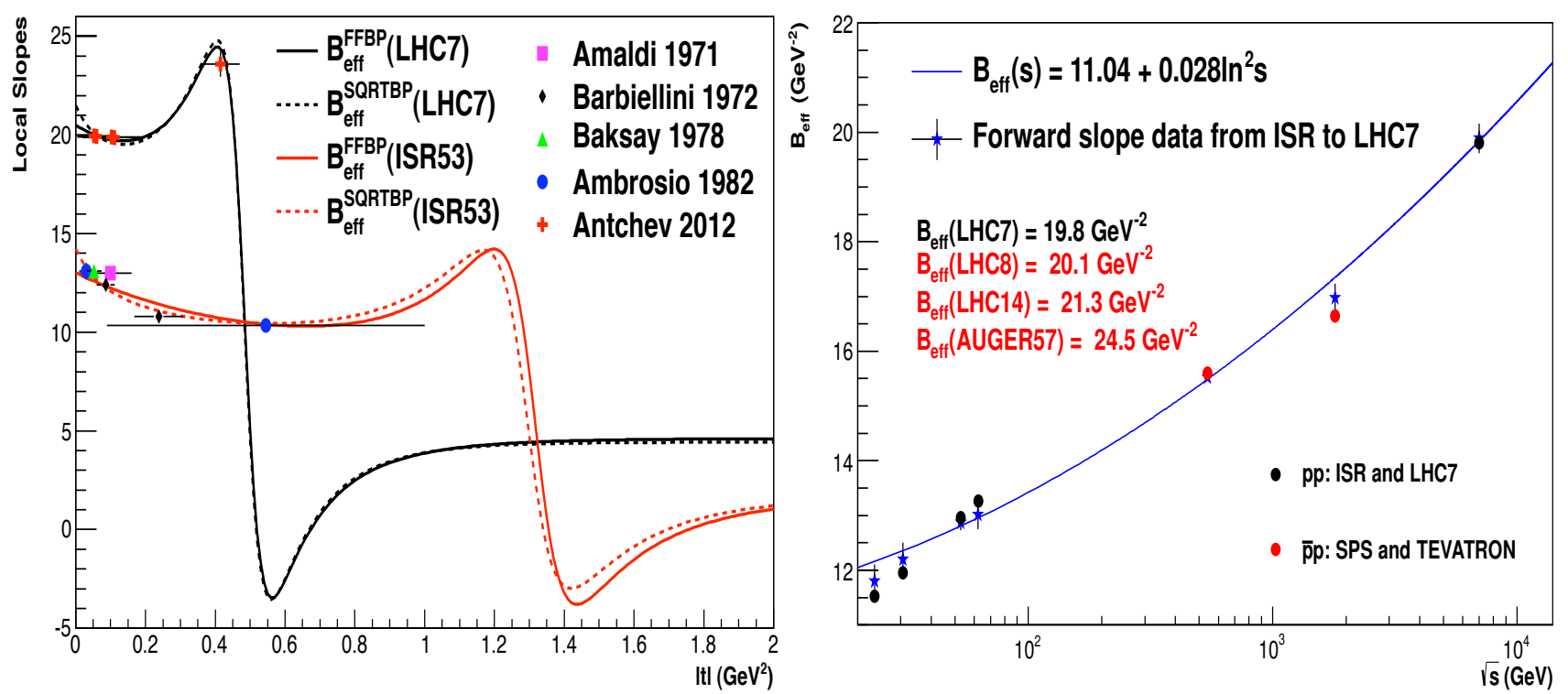

FIG. 5. At left, local and forward slopes from the two-pion threshold model (dashes, label $B_{e f f}^{S Q R T B P}$ ) and the form factor one (full line, label $B_{\text {eff }}^{F F B P}$ ) at ISR53 and LHC7. At right, the effective forward slope from both $p p$ and $\bar{p} p$ data (blue stars). Data are compared with a fit in $(\ln s)^{2}$ and from the model approximation of Eq. 15), using results from Table II for $p p$ (black dots). Red dots indicate the result for $\bar{p} p$, with the scale $t_{0}$ obtained from Fig. 3

We also notice that, from a numerical point view, while in the original BP model $B(s) \simeq B_{e f f}(s)$, in the case of $m B P 2$ the following approximation holds:

$$
B(s) \simeq B_{e f f}(s)-\frac{8}{t_{0}}
$$

as one can easily check using Eq. (14). For this model, we also show in the right panel of Fig. 5 the comparison between $B_{e f f}(s)$ from experimental results from ISR to LHC. The parametrization applied in Fig. 5 for $B_{e f f}(s)$ is inspired by the asymptotic theorems, and consistent with the result for the effective slope by Schegelski and Ryskin [19. Indeed, when fitting $B_{\text {eff }}(s)$ with an additional term with a linear $\ln s$ dependence, the respective coefficient is consistent with zero. That leads to the best fit shown in Fig. 5, with $B_{\text {eff }}(s) \sim(\ln s)^{2}$. 


\section{ASYMPTOTIC PREDICTIONS OF THE EMPIRICAL MODEL $m B P 2$}

The original BP model of Eq. (1) had purported to present a "model independent analysis of the structure in $p p$ scattering" 2. As such, and as recently pointed out by Uzhinsky and Galoyan [20, the BP parametrization does not, in itself, possess a predictive power. However, its simplicity can be exploited to make higher energy predictions. In fact, the model has the virtue of allowing a simple implementation of the asymptotic sum rules we presented in [3], and thus to obtain the asymptotic behavior of the parameters which can lead to this model predictions for the elastic differential cross-section at LHC8 and LHC14. We shall now proceed to illustrate such an asymptotic, and partly empirical, realization of the $m B P 2$ model.

This model has 6 parameters, i.e. 2 amplitudes $\sqrt{A(s)}$ and $\sqrt{C(s)}$, two slopes $B(s)$ and $D(s)$, a phase $\phi$ and a scale $t_{0}$. The fits to ISR and LHC7 data suggest $t_{0} \rightarrow 0.71 \mathrm{GeV}^{2}$ at LHC energies, thus for asymptotic predictions we fix $t_{0}$ to acquire the value of the EM form factor of the proton, i.e. $t_{0}=0.71 \mathrm{GeV}^{2}$. As for the phase $\phi$, the same fits support the approximation $\phi \sim$ constant in energy. In Regge models, the phase would be t-dependent, and, in such case, the phase, as used here in the empirical model, would represent a value averaged over the range $\Delta t$ of validity of this model.

Having thus made the ansatz that both $t_{0}$ and $\phi$ are asymptotically constant, we remain with 4 energy dependent parameters. As we shall shortly discuss in detail, to comply with asymptotic theorems $\sqrt{A}$ and $B(s)$ should have the same asymptotic behavior, namely at most like $(\ln s)^{2}$. For the slope of the second (non leading) term, an asymptotic normal Regge behavior would be the most appealing possibility. The amplitude of the second term is so far unconstrained. From the asymptotic sum rules, the amplitude $\sqrt{C(s)}$ can either have a constant or a logarithmic energy dependence. We shall now see how this behavior can be understood in more detail.

The satisfaction of the sum rules for elastic scattering at higher energies, namely, $S R_{1} \rightarrow 1$ and $S R_{0} \rightarrow 0$, is suggested by our results, presented in Table IV] Based on their saturation, we propose to make predictions concerning the energy behavior of the parameters $A(s), B(s), C(s)$ and $D(s)$. We begin with the simple BP model, which contains the asymptotics of the sum rules, since both $\gamma(s)$ and $t_{0}$ of the modified versions of the model are approximately constant in energy. The asymptotic sum rules read:

$$
\begin{aligned}
& S R_{0}=\sqrt{\frac{A(s)}{1+\hat{\rho}(s)^{2}}} \frac{\hat{\rho}(s)}{\sqrt{\pi} B(s)}-\frac{\sqrt{C(s)} \sin \phi}{\sqrt{\pi} D(s)} \rightarrow 0 \\
& S R_{1}=\sqrt{\frac{A(s)}{1+\hat{\rho}(s)^{2}}} \frac{1}{\sqrt{\pi} B(s)}+\frac{\sqrt{C(s)} \cos \phi}{\sqrt{\pi} D(s)} \rightarrow 1
\end{aligned}
$$

Since $\phi$ is approximately constant throughout the range from ISR and beyond, and if $\hat{\rho}(s) \sim 1 / \ln s$, one can then obtain the following asymptotic relationships between the parameters:

$$
\begin{array}{r}
\frac{\sqrt{A(s)}}{B(s)} \sim \frac{\sqrt{C(s)}}{D(s)} \ln s \\
\frac{\sqrt{A(s)}}{B(s)} \sim \frac{\sqrt{\pi}}{\left(1+\frac{\pi \cot \phi}{2 p \ln s}\right)} \sim \text { constant }
\end{array}
$$

We now start from the fact that to leading order in $\ln s$, the parameter $A(s) \propto \sigma_{t o t}^{2}$. The satisfaction of asymptotic theorems [21] suggests that asymptotically $\sigma_{\text {total }} \sim B(s)$, which is also in agreement with Eq. 19.

We consider here a specific realization of the Froissart-Martin bound [5, 6], namely the case of maximal energy saturation. The more general case of $\sigma_{\text {total }} \sim(\ln s)^{1 / p}$ with $1 / 2<p<1$ will be discussed elsewhere. Then,

$$
A(s) \sim(\ln s)^{4}, \quad B(s) \sim(\ln s)^{2}, \quad D(s) \sim \sqrt{C(s)} \ln s
$$

The above results are proposed in the context of the simple BP model, with 5 parameters. The Form Factor modification of Eq. (3) may introduce some changes, but, if we assume the parameter $t_{0}$ to asymptote to a constant value ( of $t_{0} \simeq 0.71 \mathrm{GeV}^{2}$ ), its introduction will not spoil the simple relations of Eqs. (18) and (19). We point out that the ansatz $B(s) \sim(\ln s)^{2}$ is asymptotically consistent with data, as discussed in [19] and seen in Fig. 5. However, at non-asymptotic energies the parameter $B(s)$ may have a slower growth.

To estimate the energy dependence of the parameters of the non-leading term, i.e. $D(s)$ and $C(s)$, is more complicate. An important consequence of Eq. $\sqrt{13}$ is that $\sqrt{C(s)}$, if at all, must increase less than $\ln s$, if both $\rho(s)$ and $\hat{\rho}(s) \sim(\ln s)^{-1}$ asymptotically. Namely 
- If the first $[$ the $\sqrt{A}]$ term in the elastic amplitude indeed represents a $C=+$ vacuum term, then

$$
\hat{\rho}(s) \rightarrow \frac{\pi}{\ln \left(s / s_{0}\right)} .
$$

- If the Froissart-Martin bound is indeed saturated, then we have the Khuri-Kinoshita theorem according to which also

$$
\rho(s) \rightarrow \frac{\pi}{\ln \left(s / s_{0}\right)} ;[\text { with the same coefficient } \pi]
$$

- If both Eq.21 and Eq.22, are simultaneously true, then we must have that

$$
[\sqrt{(C / A)}] \ln \left(s / s_{0}\right) \rightarrow 0 .
$$

- The above precludes $\sqrt{C}$ from growing asymptotically as $\ln \left(s / s_{0}\right)$, if $\sqrt{A} \sim\left[\ln \left(s / s_{o}\right)\right]^{2}$.

In the logarithmic approximations we are using here, the simplest assumption, albeit not the only one, satisfying the sum rules, the Froissart bound and the Khuri-Kinoshita theorem [22, is then

$$
D(s) \sim \ln s \quad \sqrt{C(s)} \sim \text { constant }
$$

In other terms, when the Khuri-Kinoshita asymptotic betaviour for the real part of the amplitude is satisfied, one can choose the amplitude $C(s) \rightarrow$ constant and the sum rules dictate a normal Regge-like behavior for the slope of non-leading term, $D(s)$. However there are some caveats and subtleties to be aware of:

- (i) the phenomenology presented for $p p$, and $\bar{p} p$ scattering as well, shows $\sqrt{C(s)}$ to increase very rapidly from ISR to LHC7, hence a constant behavior over this energy range is not observed (see Table II).

- (ii) For a large range of energy interval $\rho(s) \sim$ constant [average value 0.12] and thus over the same range of interval $C(s)$ may increase in order to keep $S R_{0} \sim 0$. This seems to be borne out by the phenomenology.

Thus it is quite possible that, at least in the energy range in which $\rho(s) \sim$ constant, $\sqrt{C} \sim \ln \left(s / s_{0}\right)$. Unfortunately, with present data, no unique limit can be prescribed. We shall thus resort to an empirical parameterization for $\sqrt{C(s)}$, as shown shortly below.

\section{IV.1. Phenomenological results for the parameters}

In this section we propose an empirical description of the differential elastic $p p$ cross-section to be used at LCH8 and LHC14. This parametrization follows Eq. (3) and refines the one proposed in [3, presenting an optimal description of the very small $-t$ value, in addition to the already mentioned good description of the dip and the tail.

Following the discussion in the paper, and fits to ISR and LHC7 data, we propose the following asymptotic parametrization:

$$
\begin{array}{r}
4 \sqrt{\pi A(s)}(m b)=47.8-3.8 \ln s+0.398(\ln s)^{2} \\
B(s)\left(G e V^{-2}\right)=11.04+0.028(\ln s)^{2}-\frac{8}{0.71}=-0.23+0.028(\ln s)^{2} \\
4 \sqrt{\pi C(s)}(m b)=\frac{9.6-1.8 \ln s+0.01(\ln s)^{3}}{1.2+0.001(\ln s)^{3}} \\
D(s)\left(G e V^{-2}\right)=-0.41+0.29 \ln s
\end{array}
$$

The parametrization for $C(s)$ is empirical, $\sqrt{A(s)}$ and $B(s)$ follow asymptotic maximal energy saturation behavior, $D(s)$ shows normal Regge behavior. In Fig. 6 we plots the parametrizations from Eqs. (25), (26), (27), (28), and indicate the results of the fit to the elastic differential cross-sections for $p p$ data (black dots). The red dots indicate the value of the parameters obtained when fitting $\bar{p} p$ data with the $m B P 2$ model. We leave a discussion of this model for the $\bar{p} p$ case to a forthcoming paper. Notice that $\bar{p} p$ data were not used to determine the parametrization given in Eqs.25, 26, 227, ,28). 

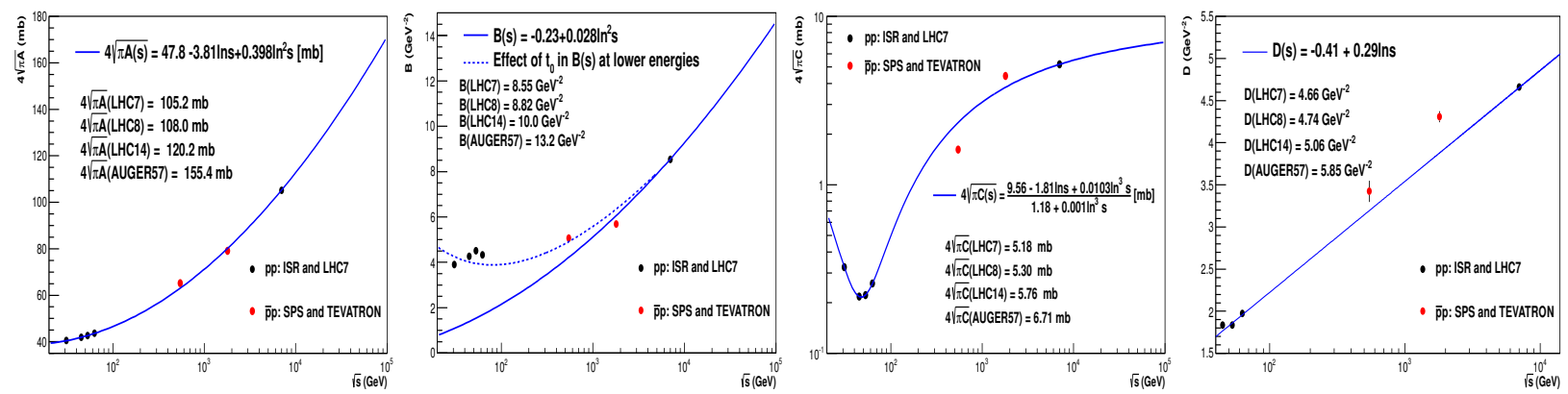

FIG. 6. Energy behavior of the model parameters $\sqrt{A(s)}, B(s), \sqrt{C(s)}, D(s)$ described in the text. Black dots correspond to parameter values determined by fit to $p p$ scattering data. Red dots correspond to the parameter values fitting $\bar{p} p$ data, and were not used to determine the parametrization given in Eqs. 225, 26), 27), 28.

\section{IV.2. The position of the dip}

Although the phase $\phi$ is consistent with a constant as the energy increases, its value fluctuates. In the range $\sqrt{s}=53-7000 \mathrm{GeV}$, the fits for $p p$ and $\bar{p} p$ indicate $\phi \simeq 2.7-2.8 \mathrm{rad}$. We note that the value used for $\phi$ influences the position and depth of the dip. In order to choose a value for $\phi$, we then study how the dip moves as a function of energy. The simplest asymptotic assumption for the dip position as a function of energy is to assume geometrical scaling, namely $t \sigma_{\text {total }} \sim$ constant. In the maximal saturation model, in which $\sigma_{\text {total }} \sim(\ln s)^{2}$, one can then parametrize the dip position as

$$
t_{d i p}=-\frac{a}{1+b(\ln s)^{2}}
$$

In Fig. 7 we compare data for the position of the dip in $p p$ scattering with a parametrization obtained from Eq. 29 and with other predictions from amplitudes obeying geometrical scaling as discussed in [23. A linear logarithmic fit is also shown for comparison.

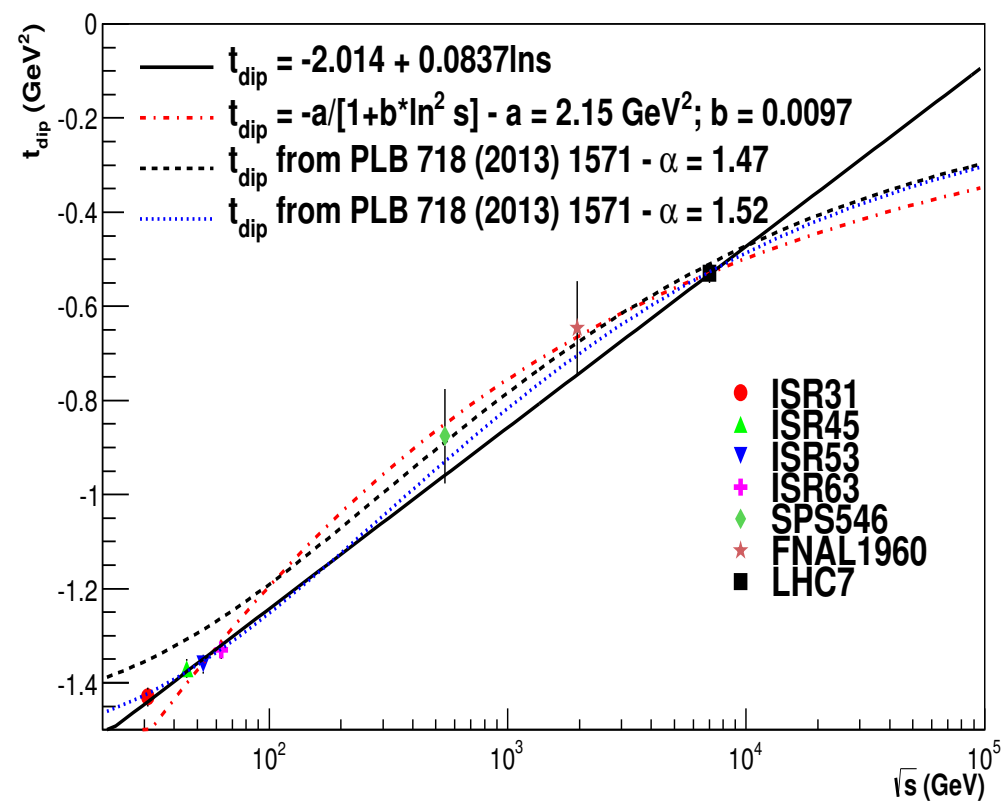

FIG. 7. Experimental values for the position of the dip in $p p$ and $\bar{p} p$ elastic scattering vs. models suggested by geometrical scaling [23] or a simple logarithmic energy rise.

Using these different possibilities, one can calculate the position of the dip at LHC8 and LHC14, as shown in Table V] In this table, GS1 refer to the parametrization of Eq. 29, GS2 and GS3 to different applications of the 
geometrical scaling model of Ref. [23. The geometrical scaling values are in good agreement with recent predictions for the dip position at LHC14 from [24, 25].

TABLE V. Dip position from $\sqrt{s}=8 \mathrm{TeV}$ onwards as predicted by geometrical scaling models and simple linear logarithmic evolution.

\begin{tabular}{ccccc}
\hline \hline$\sqrt{s}(\mathrm{TeV})$ & $|t|_{\text {dip }}^{L I N}$ & $|t|_{\text {dip }}^{G S 1}$ & $|t|_{\text {dip }}^{G S 2}$ & $|t|_{\text {dip }}^{G S 3}$ \\
\hline 8 & 0.510 & 0.518 & 0.495 & 0.511 \\
\hline 14 & 0.417 & 0.471 & 0.439 & 0.452 \\
\hline \hline
\end{tabular}

\section{PREDICTIONS FOR LHC8 AND LHC14 AND THE BLACK DISK LIMIT}

We are now in a position to predict the $t$-dependence of the elastic differential cross-section in $p p$ scattering at higher LHC energies, using the empirical asymptotic model described in the previous section. In Fig 8 we show these predictions for $p p$ elastic differential cross-sections at LHC8 and LHC14.
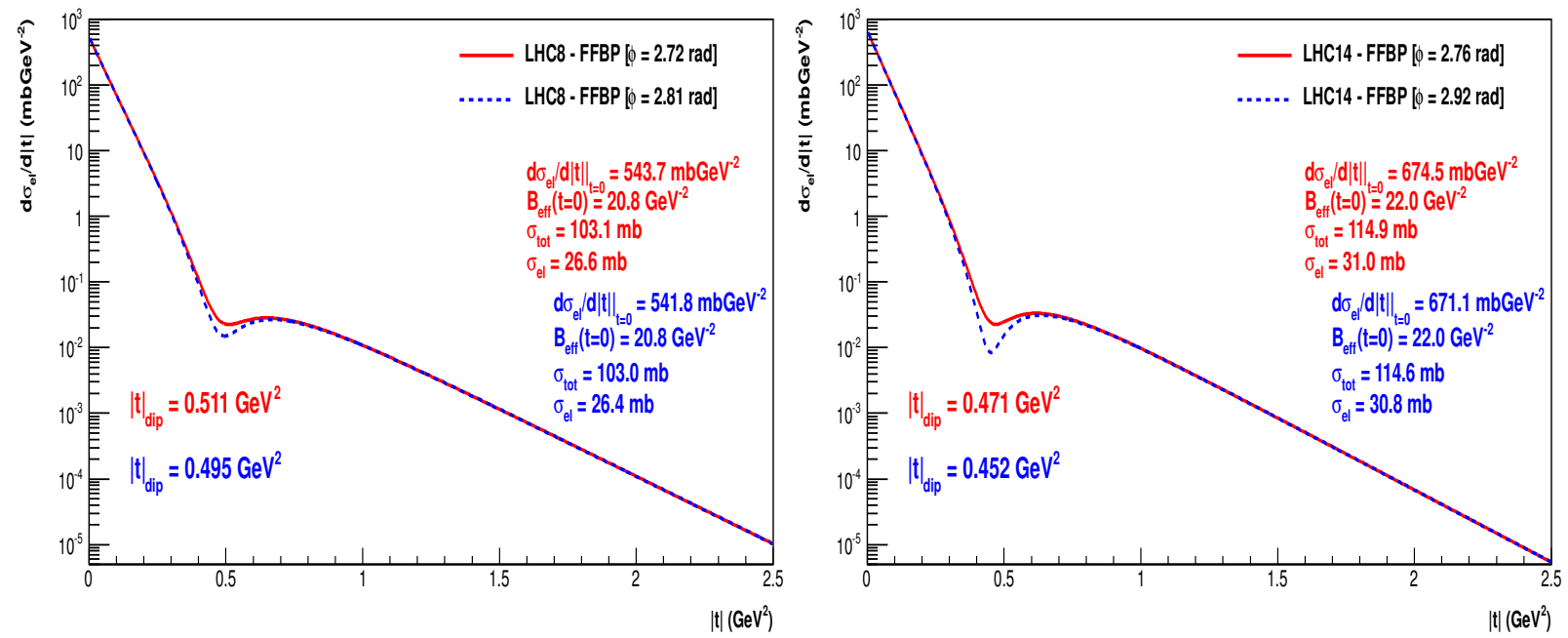

FIG. 8. $m B P 2$ model predictions for the differential elastic cross section at LHC8 and LHC14 in a maximal energy saturation model, $\sigma_{\text {total }} \sim(\ln s)^{2}$.

This model does not include a second dip, or a wiggle, as in many eikonal models, such as for instance seen in [26]. On the other hand, at present, at LHC7, in the interval $0<-t<2.5 \mathrm{GeV}^{2} \mathrm{TOTEM}$ data do not allow to establish the presence of a second dip or wiggle. Finally, the dotted and full line correspond to different values of the phase $\phi$ and the figures confirm the sensitivity of the dip depth and position to the chosen value for the phase $\phi$. We now turn to higher energies and consider one favorite test of asymptotia, namely the black disk limit. As also noticed in [27, present data from LHC7 indicate that we are still far from this limit. The question is : how far?

Using the energy parametrization discussed in the previous section, an approximately constant scale $t_{0}$ and a band of values for $\phi$, we obtain the result shown in Fig.9. We notice that this ratio is in agreement with AUGER results 28 . Moreover, the asymptotic behavior is dictated by the Sum Rules, which reinforcing the condition of total absorption of partial waves, lead to the saturation of the black disk limit, i.e. $R_{e l} \rightarrow 1 / 2$ as $s \rightarrow \infty$. From the parameters presented in Table $\mathrm{VI}$. we estimate that $R_{e l} \simeq 1 / 2$ at $\sqrt{s} \simeq 10^{10} \mathrm{GeV}$ (corresponding to the energy in the lab frame 
$\left.E \simeq 10^{20} \mathrm{GeV}\right)$, i.e. at energies typically larger than the Planck scale.

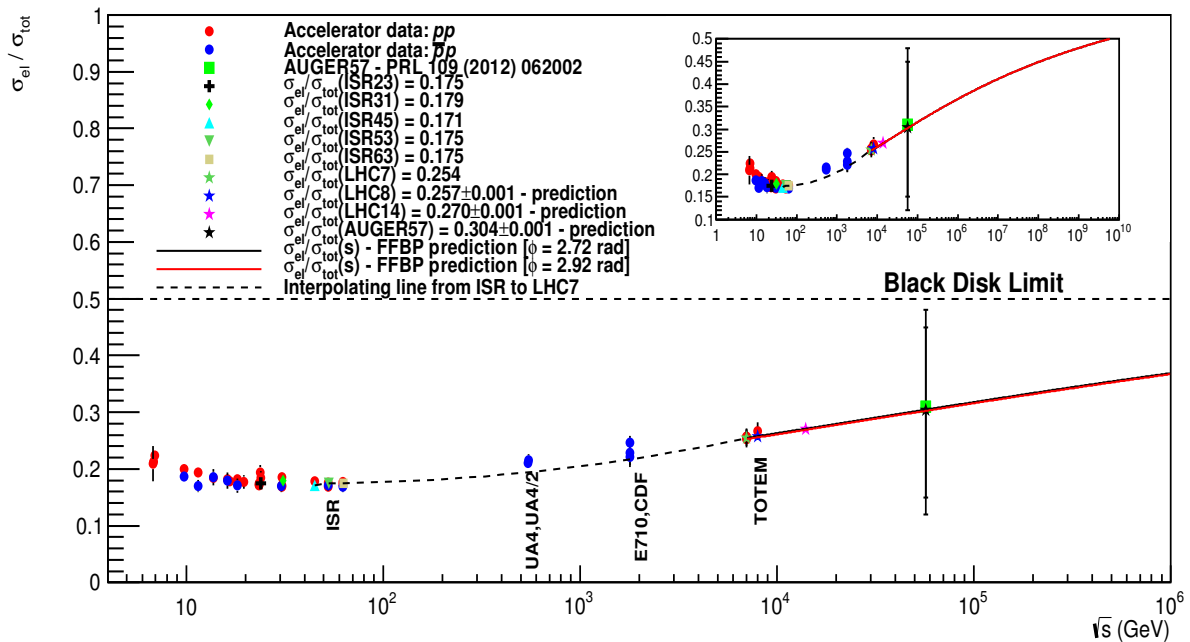

FIG. 9. Experimental data from accelerators for the ratio $R_{e l}=\sigma_{\text {elastic }} / \sigma_{\text {total }}$ as compiled from [1, 29, 30] and this model expectations. The AUGER datum has been extracted from the ratio $\sigma_{\text {inel }} / \sigma_{\text {total }}$ at $\sqrt{s}=57 \mathrm{TeV}$, as coming from estimates presented in [28]. For this point, the inner bars comprise only statistical and systematic uncertainties combined quadratically and the outer bars incorporate the total uncertainty, with errors from Glauber calculations also summed in quadrature. Inner bars: $R_{e l}^{\text {stat }+ \text { sys }}(57 \mathrm{TeV})=0.31_{-0.16}^{+0.14}$, outer bars: $R_{e l}^{\text {stat }+ \text { sys }+G l a u b e r}(57 \mathrm{TeV})=0.31_{-0.19}^{+0.17}$.

As expected, the ratio $\mathcal{R}_{e l}$ is less sensitive to variations in $\phi$, since the contribution arising from the dip region to the integrated elastic cross section is minimal. Therefore, notwithstanding the observable effect in the elastic differential cross section, shown in Fig. 8, the predictions of this model for different values of $\phi$ lead to practically overlapping curves.

TABLE VI. Values of $m B P 2$ parameters used in the predictions at LHC8, LHC14 and AUGER57 and the ratio $\mathcal{R}_{e l}$ at each c.m. energy. In all cases the values of $t_{0}$ has been frozen at $0.71 \mathrm{GeV}^{2}$ and bands for $\phi$ were considered. These bands determine the uncertainty in the predictions for the ratio.

\begin{tabular}{c|c|c|c|c|c||c}
\hline \hline$\sqrt{s}(\mathrm{TeV})$ & $A\left(\mathrm{mbGeV}^{-2}\right)$ & $B\left(\mathrm{GeV}^{-2}\right)$ & $C\left(\mathrm{mbGeV}^{-2}\right)$ & $D\left(\mathrm{GeV}^{-2}\right)$ & $\phi(\mathrm{rad})$ & $\sigma_{\text {el }} / \sigma_{\text {tot }}$ \\
\hline 8.0 & 596 & 8.8 & 1.44 & 4.7 & $2.72-2.81$ & $0.257 \pm 0.001$ \\
\hline 14 & 739 & 10.0 & 1.70 & 5.1 & $2.76-2.92$ & $0.270 \pm 0.001$ \\
\hline 57 & 1233 & 13.2 & 2.30 & 5.9 & $2.72-2.92$ & $0.304 \pm 0.001$ \\
\hline \hline
\end{tabular}

\section{CONCLUSIONS}

We have shown that the $p p$ differential elastic cross-section in the range measured by the TOTEM experiment at LHC can be parametrized through two exponentials and a phase, provided the first term is modified by a multiplicative factor to optimize the description of the forward peak. Two different modifications are proposed. For the model with a proton form factor to modify the $-t \simeq 0$ behavior, we extract predictions at LHC8 and LHC14, and calculate the ratio of the elastic to the total cross-section up to and beyond $\sqrt{s}=57 \mathrm{TeV}$.

The parametrization of $p p$ elastic cross-section data presented in this paper is not meant to be exact, rather to indicate how to break up the amplitude in a set of building blocks, and apply this dissection to the data as the energy increases. This parameterization addresses the following basic elements:

- the value of the differential cross-section at $t=0$, namely the optical point value

- a rapid decrease, characterized by a slope, which, between $-t=0$ and the dip, is not a constant 
- the occurrence of a dip in $p p$ at all energies from ISR to LHC

- an exponential decrease after the dip, with a non-leading slope and an amplitude much smaller than before the dip.

This behavior is described by an empirical model, with two amplitudes, two different slopes, a phase and the proton form factor to multiply the amplitudes. This empirical model might help us to understand the elastic $p p$ differential cross-section [31. It describes the data well and, as such, can be used by model builders and experimentalists alike.

The interpretation of the model is in parts straightforward, but not completely. In our previous analysis of TOTEM data for the elastic differential cross-section [3], we have commented on the physical meaning of the model. Our considerations were that the two terms in the amplitude receive contributions from different charge conjugation processes, the first term purely from $C=+1$, the second non-leading term has contributions from both $C= \pm 1$ terms, which, at high energy, render $\phi \neq \pi, \pi / 2$. The energy behavior of the leading amplitude $A(s)$ is consistent with many eikonal models, but the exponential behavior in the momentum transfer before and after the dip is not, and it is probably due to rescattering effects in the final state. On the other hand, the modification of the model with a form factor which reproduces the proton electromagnetic form factor at high energy, suggests the need to include rescattering effects within each colliding hadron, namely the probability that the proton does not break up as the momentum transfer increases.

\section{ACKNOWLEDGMENTS}

We thank L. Jenkovszky, M. J. Menon and J. Soffer for useful discussions. AG acknowledges partial support by Spanish MEC (FPA2010-16696, AIC-D-2011-0818) and by Junta de Andalucia (FQM 03048, FQM 6552, FQM 101). DAF acknowledges the São Paulo Research Foundation (FAPESP) for financial support (contract: 2012/12908-4).

\section{Appendix A: Two-pion threshold effects on the BP model: $m B P 1$}

We discuss here a model where the very small $-t$ behavior is influenced by the nearest $t$-channel singularity of the scattering amplitude. In this model, which we call $m B P 1$,

$$
\mathcal{A}(s, t)=i\left[\sqrt{A(s)} e^{B(s) t / 2} G(s, t)+e^{i \phi} \sqrt{C(s)} e^{D(s) t / 2}\right]
$$

with $G(s, 0)=1$ in order not to spoil the good description of the dip by Eq. (1), as discussed in the text. Such factor would arise from the contribution of the two-pion loop in the Pomeron trajectory as originally proposed in [9] and [8], and more recently discussed by Khoze, Martin and Ryskin [10 and Jenkovszky 11, 32. In particular $\alpha_{P}(t)$, at very small $t$, should include a square root singularity at $t=4 \mu^{2}$, with $\mu$ the pion mass. Mindful of such possibilities, we have applied the following correction to the first term of Eq. (1), namely we shall use

$$
G(s, t)=e^{-\gamma(s)\left(\sqrt{4 \mu^{2}-t}-2 \mu\right)},
$$

with $\gamma(s)$ a free parameter. Being applied to the near-forward region, such term shall influence the small $|t|$ behavior of elastic differential cross section, producing a changed curvature in the effective slope $B_{\text {eff }}(s, t)$ in this region. The original expressions for the total cross section and the optical point remain unchanged, but the modification of the model of Eq. (1) given by Eqs. A1, A2 introduces an additional $t$-dependence in the first term, through a square root, and hence a sixth parameter. Using the modified BP model of Eqs. A1, A2 (henceforth called $m B P 1$ ), we update our fits [3] to LHC7 data samples as well as to the ISR data sets in the full range for $p p$ data with $\sqrt{s}=(23 \div 63)$ GeV, as displayed in Table VII and Fig. 10. ISR data sets used in the fits comprise the data collection by Amaldi and Schubert [12 and all experimental information available from 1980 onwards [13 16. This table shows that this modification gives an acceptable statistical description from the optical point to the full $|t|$ range. 
TABLE VII. Values of free fit parameter $A, B, C, D, \gamma$ and $\phi$ at each energy analyzed. $A$ and $C$ are expressed in units mbGeV ${ }^{-2}$, $B$ and $D$ in units $\mathrm{GeV}^{-2}, \gamma$ in units $\mathrm{GeV}^{-1}$ and $\phi$ in radians.

\begin{tabular}{|c|c|c|c|c|c|c||c|}
\hline \hline$\sqrt{s}(\mathrm{GeV})$ & $A$ & $B$ & $C\left(\times 10^{-3}\right)$ & $D$ & $\gamma$ & $\phi$ & $\frac{\chi^{2}}{\mathrm{DOF}}$ \\
\hline 24 & $82.8 \pm 1.0$ & $6.3 \pm 0.1$ & $2.3 \pm 0.2$ & $1.79 \pm 0.04$ & $2.15 \pm 0.07$ & $2.94 \pm 0.01$ & $\frac{200}{134-6}=1.1$ \\
\hline 31 & $85.1 \pm 0.2$ & $6.99 \pm 0.06$ & $1.9 \pm 0.1$ & $1.79 \pm 0.02$ & $1.79 \pm 0.03$ & $3.02 \pm 0.01$ & $\frac{310}{206-6}=1.6$ \\
\hline 45 & $91.5 \pm 0.2$ & $7.51 \pm 0.05$ & $1.18 \pm 0.06$ & $1.62 \pm 0.02$ & $1.92 \pm 0.03$ & $2.73 \pm 0.02$ & $\frac{801}{207-6}=4.0$ \\
\hline 53 & $94.6 \pm 0.1$ & $7.78 \pm 0.05$ & $1.49 \pm 0.05$ & $1.70 \pm 0.01$ & $1.79 \pm 0.02$ & $2.68 \pm 0.01$ & $\frac{1490}{319-6}=4.8$ \\
\hline 63 & $98.5 \pm 0.2$ & $7.98 \pm 0.09$ & $1.7 \pm 0.1$ & $1.75 \pm 0.03$ & $1.74 \pm 0.04$ & $2.75 \pm 0.03$ & $\frac{332}{165-6}=2.1$ \\
\hline 7000 & $565 \pm 2$ & $13.7 \pm 0.2$ & $970 \pm 40$ & $4.43 \pm 0.03$ & $2.01 \pm 0.06$ & $2.703 \pm 0.007$ & $\frac{497}{161-6}=3.2$ \\
\hline \hline
\end{tabular}
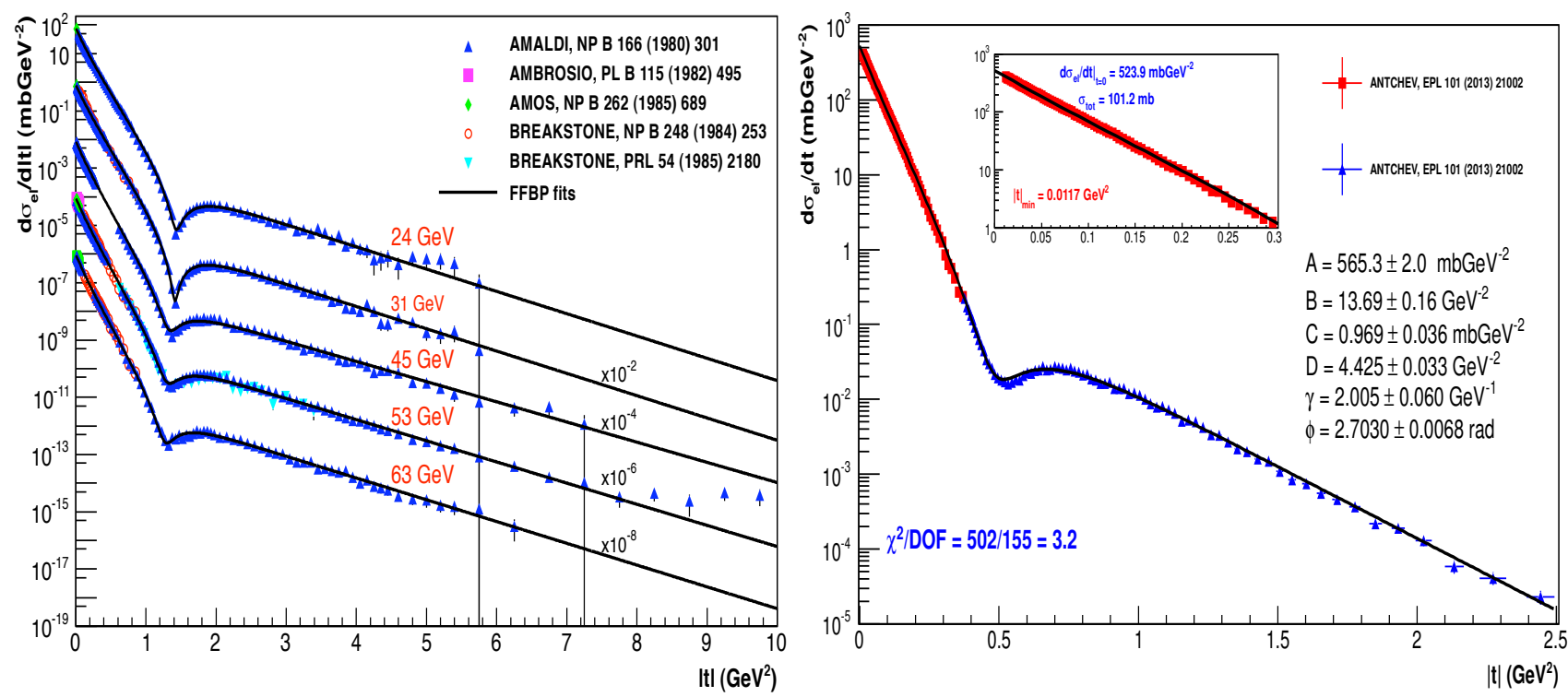

FIG. 10. Fits to the ISR and LHC7 data sets with model $m B P 1$.

In Fig. 11 we present the energy dependence of fit parameters for $m B P 1$ model . The continuous (dotted) lines in these figures are computer parametrizations drawn to guide the eye.
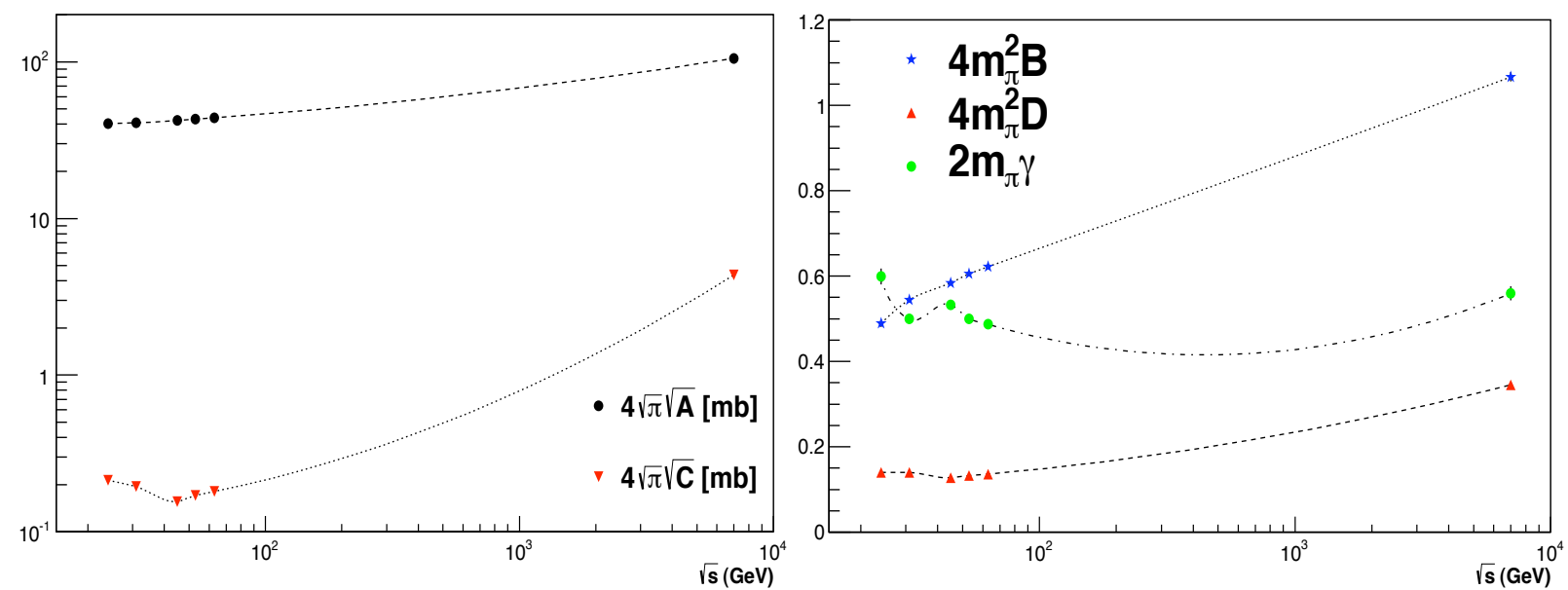

FIG. 11. Energy behavior of parameters from the $m B P 1$ model. 
We now make two comments. Firstly, the square root factor is only used for the first term of the BP amplitude, as this factor comes from the contribution of the pion loop to the leading vacuum term and it may not be present for the second non-leading term, which, for a generic $\phi$, has contributions also from $C=-1$ processes. The second comment derives from an inspection of Table VII and the energy dependence of the parameter $\gamma(s)$. This energy dependence, displayed in Fig. 11 from ISR to LHC7, shows a very slow increase, even compatible with a constant in energy, shedding doubt on a straightforward interpretation of this factor in terms of a small $t$ contribution to the Pomeron trajectory.

The elastic cross section for this model from Eqs. A1, A2 is obtained as

$$
\sigma_{e l}(s)=\int_{-\infty}^{0} d t|\mathcal{A}(s, t)|^{2}=A e^{4 m_{\pi} \gamma} \mathcal{I}_{1}+\frac{C}{D}+2 \sqrt{A C} e^{2 m_{\pi} \gamma} \cos \phi \mathcal{I}_{3},
$$

where the integrals $\mathcal{I}_{1}$ and $\mathcal{I}_{3}$ are given as:

$$
\begin{aligned}
& \mathcal{I}_{1}=\int_{-\infty}^{0} d t e^{B t-2 \gamma \sqrt{4 m_{\pi}^{2}-t}}, \\
& \mathcal{I}_{3}=\int_{-\infty}^{0} d t e^{(B+D) t / 2-\gamma \sqrt{4 m_{\pi}^{2}-t}} .
\end{aligned}
$$

An analytical evaluation can be obtained, used the result:

$$
\mathcal{I}(\alpha, \beta, \delta) \equiv \int_{-\infty}^{0} d t e^{\alpha t-\beta \sqrt{\delta^{2}-t}}=\frac{1}{\alpha} e^{-\delta \beta}-\frac{\beta \sqrt{\pi}}{2 \alpha^{3 / 2}} \operatorname{Erfc}[\sqrt{\alpha}(\delta+\beta / 2 \alpha)] e^{\alpha \delta^{2}+\beta^{2} / 4 \alpha},
$$

where $\operatorname{Erfc}(x)=\frac{2}{\sqrt{\pi}} \int_{x}^{\infty} e^{-y^{2}} d y$ denotes the complementary error function. Thus, from Eqs. A4 - A6 it follows that:

$$
\begin{aligned}
\sigma_{e l}(s) & =\frac{A}{B}+\frac{C}{D}+\frac{4 \sqrt{A C}}{(B+D)} \cos \phi-\sqrt{\pi} \frac{A \gamma}{B^{3 / 2}} \operatorname{Erfc}\left[\sqrt{B}\left(2 m_{\pi}+\frac{\gamma}{B}\right)\right] e^{4 m_{\pi}^{2}\left(B+\gamma / m_{\pi}\right)+\gamma^{2} / B} \\
& -\sqrt{8 \pi} \frac{\sqrt{A C} \gamma \cos \phi}{(B+D)^{3 / 2}} \operatorname{Erfc}\left[\sqrt{\frac{B+D}{2}}\left(2 m_{\pi}+\frac{\gamma}{B+D}\right)\right] e^{2 m_{\pi}^{2}\left(B+D+\gamma / m_{\pi}\right)+\gamma^{2} / 2(B+D)}
\end{aligned}
$$

In the above expression one can see that the contributions with positive sign come from the simple BP amplitude, as one can be easily checked by taking the limit $\gamma \rightarrow 0$. Thus, the presence of negative terms in Eq. (A7), being due to $G(s, t)$, reflects the importance of modifying the first term of the original BP amplitude.

The sum rules for the elastic amplitude presented in Ref. [3] can be applied to this model, and used to to check the saturation of the elastic amplitude at LHC energies. One has:

$$
\begin{aligned}
& S R_{1}=\frac{1}{\sqrt{\pi} B} \sqrt{\frac{A}{1+\hat{\rho}^{2}}}+\frac{\sqrt{C}}{\sqrt{\pi} D} \cos \phi-\sqrt{\frac{\pi}{2} \frac{A}{1+\hat{\rho}^{2}}} \frac{\gamma}{B^{3 / 2}} \operatorname{Erfc}\left[\sqrt{\frac{B}{2}}\left(2 m_{\pi}+\frac{\gamma}{B}\right)\right] e^{2 m_{\pi}^{2}\left(B+\gamma / m_{\pi}\right)+\gamma^{2} / 2 B} ; \\
& S R_{0}=\frac{\hat{\rho}}{\sqrt{\pi} B} \sqrt{\frac{A}{1+\hat{\rho}^{2}}}-\frac{\sqrt{C}}{\sqrt{\pi} D} \sin \phi-\hat{\rho} \sqrt{\frac{\pi}{2} \frac{A}{1+\hat{\rho}^{2}}} \frac{\gamma}{B^{3 / 2}} \operatorname{Erfc}\left[\sqrt{\frac{B}{2}}\left(2 m_{\pi}+\frac{\gamma}{B}\right)\right] e^{2 m_{\pi}^{2}\left(B+\gamma / m_{\pi}\right)+\gamma^{2} / 2 B} .
\end{aligned}
$$

As above for the elastic cross section, the first two terms come from the original BP amplitude and the input $G(s, t)$ produce the last term.

\section{Appendix B: Other form factor modifications of the Barger and Phillips model}

We examine here two more possible modifications of the Barger and Phillips model, complementary to the form factor modification of the first term, presented in the text:

- the entire BP amplitude is multiplied by a factor

$$
F_{P}^{2}=\frac{1}{\left(1-t / t_{0}\right)^{4}}
$$


with $t_{0}$ a free parameter, namely

$$
\mathcal{A}(s, t)=i F_{P}^{2}(t)\left[\sqrt{A(s)} e^{B(s) t / 2}+e^{i \phi(s)} \sqrt{C(s)} e^{D(s) t / 2}\right]
$$

- both terms of the BP amplitude are multiplied by a form factor (squared), but with difference scales, $t_{0}$ and $t_{O}$, namely

$$
\mathcal{A}(s, t)=i\left[F_{P}^{2}(t) \sqrt{A(s)} e^{B(s) t / 2}+e^{i \phi(s)} F_{O}^{2}(t) \sqrt{C(s)} e^{D(s) t / 2}\right]
$$

with

$$
F_{O}^{2}=\frac{1}{\left(1-t / t_{O}\right)^{4}}
$$

with $t_{0, O}$ free parameters.

We show the results of the fit in Fig. 12 .
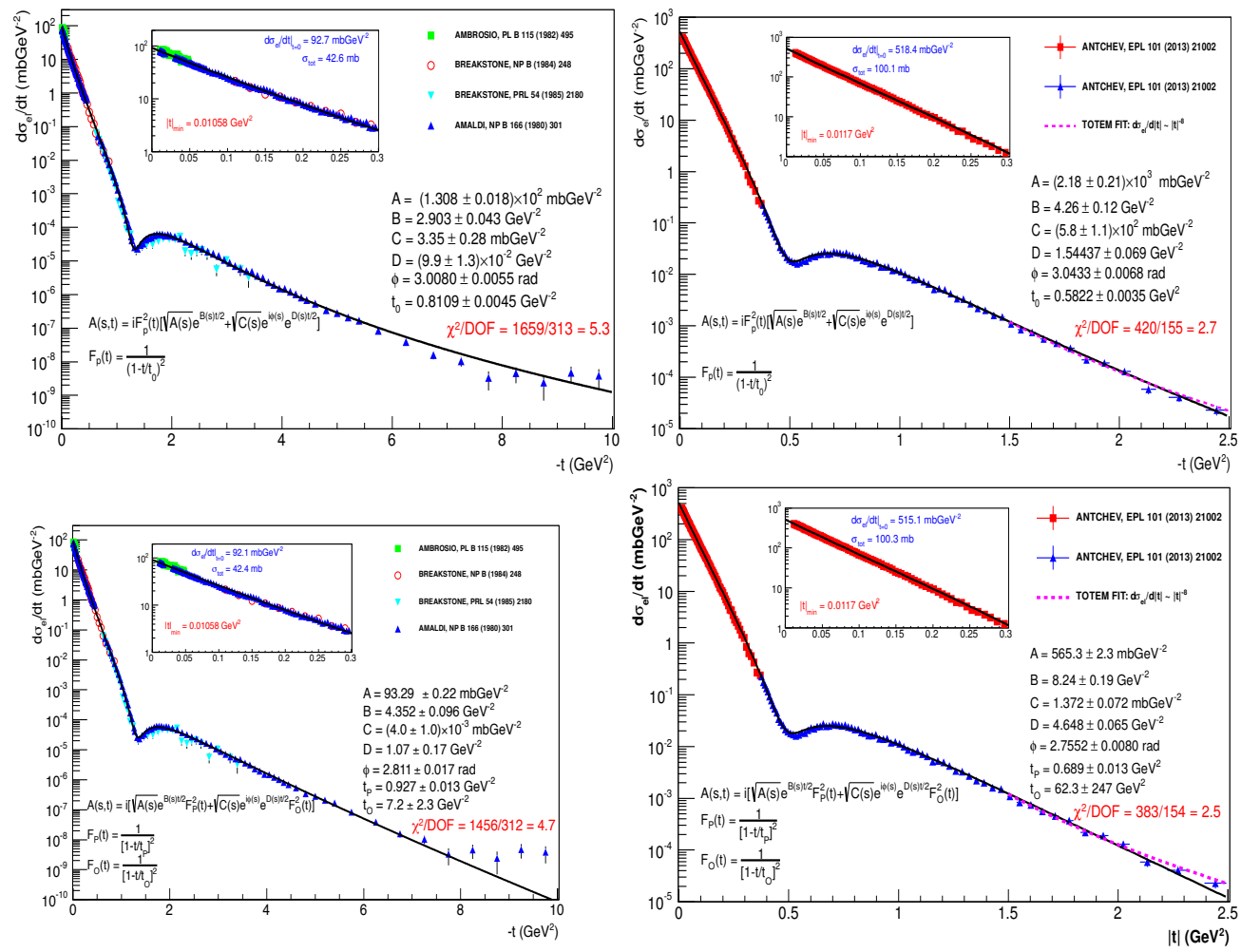

FIG. 12. Fits to ISR53 and LHC7 data sets with the two other modified BP models described by Eqs. (B2) and and (B3), with ISR fits on the left hand side and LHC fits on the right hand side. Top row: BP amplitude multiplied by an overall form factor like term. Bottom row: the two terms in BP amplitude are multiplied by form factors with different scales.

An inspection of these fits indicates that an overall multiplicative factor, corresponding to the first top plots, is least favored of the above two possibilities (and less favored than the one chosen in the text, $m B P 2$ ). From the point of view of the $\chi^{2}$, the fits do not favor the second possibility relative to the choice $m B P 2$, discussed in the text: multiplying both terms by form factors with different scales or only the first term as in $m B P 2$, gives an equally good fit, both at ISR and at LHC. However, we notice a problem with the fits of the bottom figures, when the two terms are each multiplied by a different factor, namely these fits are quite insensitive to the second scale. Phenomenologically therefore, this possibility is not particularly useful, albeit it could be further studied. 


\section{Appendix C: Impact parameter structure in the modified models}

Besides the sum rules, the impact parameter structure of models $m B P 1$ and $m B P 2$ provides us useful information about unitarity saturation. From our fits with both models, we extract the elastic profile, through the Hankel transform of the amplitude (A1):

$$
\tilde{\mathcal{A}}(s, b)=-i \int_{0}^{\infty} q d q J_{0}(q b) \mathcal{A}(s, t) .
$$

On the one hand, the dominant contribution comes from the real part, which assume distinct forms for models $m B P 1$ and $m B P 2$ :

$$
\begin{aligned}
& \tilde{\mathcal{A}}_{R}^{m B P 1}(s, b)=\sqrt{A} e^{2 m_{\pi} \gamma} \mathcal{J}(s, b)+\frac{\sqrt{C}}{D} e^{-b^{2} / 2 D} \cos \phi \\
& \mathcal{A}_{R}^{m B P 2}(s, b)=\sqrt{A} t_{0}^{4} \mathcal{K}(s, b)+\frac{\sqrt{C}}{D} e^{-\frac{b^{2}}{2 D}} \cos \phi
\end{aligned}
$$

where the integrals $\mathcal{J}(s, b)$ and $\mathcal{K}(s, b)$ are given as

$$
\begin{aligned}
& \mathcal{J}(s, b)=\int_{0}^{\infty} q d q J_{0}(q b) e^{-B q^{2} / 2-\gamma \sqrt{4 m_{\pi}^{2}+q^{2}}} \\
& \mathcal{K}(s, b)=\int_{0}^{\infty} q d q J_{0}(q b) \frac{e^{-B q^{2} / 2}}{\left(t_{0}+q^{2}\right)^{4}}
\end{aligned}
$$

On the other, the imaginary part turns out to be the same:

$$
\mathcal{A}_{I}^{m B P 1, m B P^{2}}(s, b)=\frac{\sqrt{C}}{D} e^{-\frac{b^{2}}{2 D}} \sin \phi .
$$

Unfortunately, due to the introduction of corrections into the first term of original BP parametrization, the integrals C4, C5 can no longer be solved analitically. Therefore, we perform numerical evaluations of such integrals. In Fig. 13 we present these calculations and the energy evolution of the elastic $b$-distributions, following from Eqs. C2 C6 , from ISR energies to LHC7.
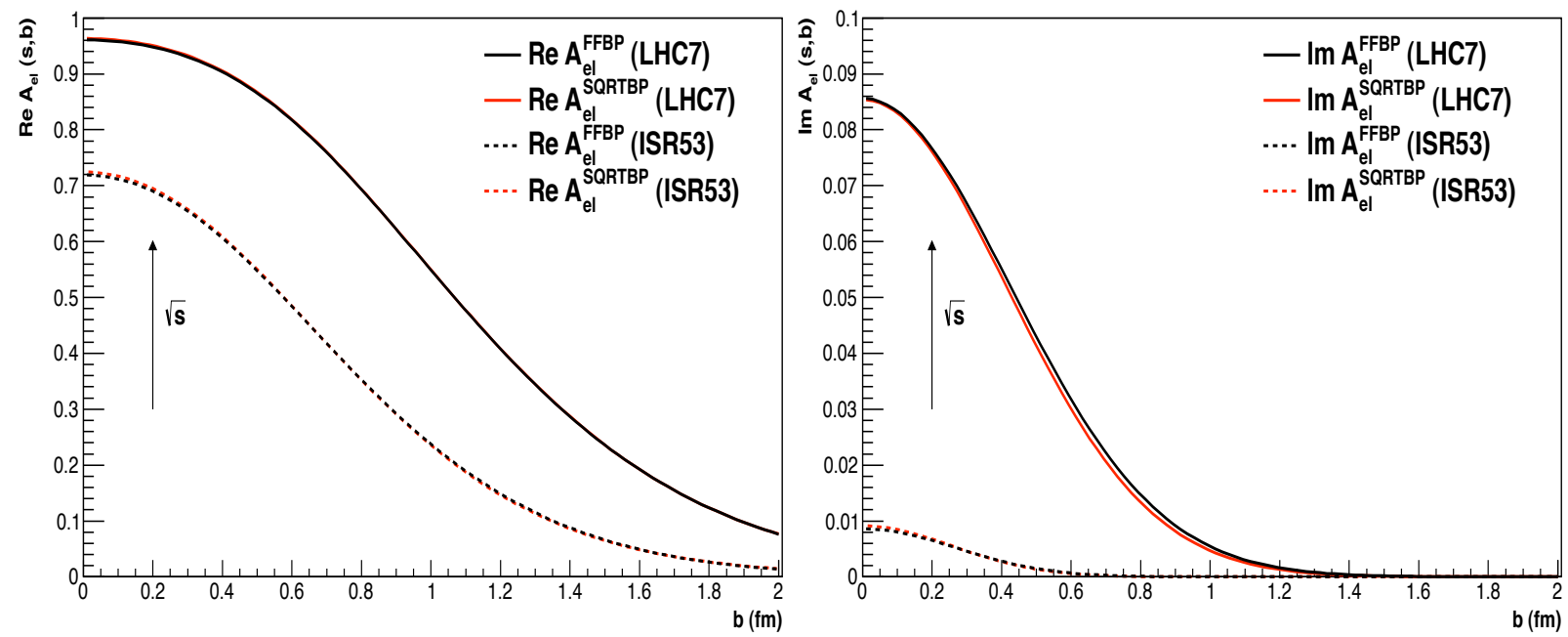

FIG. 13. Energy evolution of profile functions (real and imaginary) from ISR to LHC7.

[1] G. Antchev et al. (TOTEM Collaboration), Europhys.Lett. 101, 21002 (2013) 
[2] R. J. N. Phillips and V. D. Barger, Phys.Lett. B46, 412 (1973).

[3] A. Grau, S. Pacetti, G. Pancheri, and Y. N. Srivastava, Phys.Lett. B714, 70 (2012), arXiv:1206.1076 [hep-ph].

[4] G. Antchev et al. (TOTEM Collaboration), Europhys.Lett. 95, 41001 (2011), arXiv:1110.1385 [hep-ex]]

[5] M. Froissart, Phys.Rev. 123, 1053 (1961).

[6] A. Martin, Phys.Rev. 129, 1432 (1963).

[7] We note here that an error had occurred in [3] when fitting ISR data at $\sqrt{s}=53 \mathrm{GeV}$, with inclusion of $63 \mathrm{GeV}$ data in the fit. Conclusions, however, remain unchanged.

[8] G. Cohen-Tannoudji, V. V. Ilyin, and L. L. Jenkovszky, Lett.Nuovo Cim. 5S2, 957 (1972).

[9] A. A. Anselm and V. N. Gribov, Phys.Lett. B40, 487 (1972).

[10] V. A. Khoze, A. D. Martin, and M. G. Ryskin, Eur.Phys.J. C18, 167 (2000), arXiv:hep-ph/0007359 [hep-ph].

[11] R. Fiore, L. L. Jenkovszky, R. Orava, E. Predazzi, A. Prokudin, et al., Int.J.Mod.Phys. A24, 2551 (2009) arXiv:0810.2902 [hep-ph].

[12] U. Amaldi and K. R. Schubert, Nucl.Phys. B166, 301 (1980)

[13] N. A. Amos, M. Block, G. Bobbink, M. Botje, D. Favart, et al., Nucl.Phys. B262, 689 (1985)

[14] A. Breakstone et al. (AMES-BOLOGNA-CERN-DORTMUND-HEIDELBERG-WARSAW COLLABORATION), Nucl.Phys. B248, 253 (1984).

[15] A. Breakstone, H. Crawley, G. Dallavalle, K. Doroba, D. Drijard, et al., Phys.Rev.Lett. 54, 2180 (1985)

[16] M. Ambrosio et al. (CERN-Naples-Pisa-Stony Brook Collaboration), Phys.Lett. B115, 495 (1982)

[17] R. M. Godbole, A. Grau, G. Pancheri, and Y. N. Srivastava, Phys. Rev. D72, 076001 (2005), arXiv:hep-ph/0408355.

[18] A. Grau, R. M. Godbole, G. Pancheri, and Y. N. Srivastava, Phys.Lett. B682, 55 (2009), arXiv:0908.1426 [hep-ph]

[19] V. A. Schegelsky and M. G. Ryskin, Phys.Rev. D85, 094024 (2012), arXiv:1112.3243 [hep-ph].

[20] V. Uzhinsky and A. Galoyan, arXiv:1210.7338 [hep-ph] (2012), arXiv:1210.7338 [hep-ph]

[21] M. M. Block and R. N. Cahn, Rev. Mod. Phys. 57, 563 (1985).

[22] N. N. Khuri and T. Kinoshita, Phys.Rev. 137, B720 (1965).

[23] I. Bautista and J. Dias de Deus, Phys.Lett. B718, 1571 (2013), arXiv:1212.1764 [nucl-th].

[24] C. Bourrely, J. M. Myers, J. u. Soffer, and T. T. Wu, Phys.Rev. D85, 096009 (2012), arXiv:1202.3611 [hep-ph]

[25] E. Ferreira, T. Kodama, and A. Kohara, Eur.Phys.J. C73, 2326 (2013), arXiv:1212.3652 [hep-ph]

[26] M. M. Block and F. Halzen, Phys.Rev. D86, 014006 (2012), arXiv:1205.5514 [hep-ph].

[27] M. M. Block and F. Halzen, Phys.Rev. D86, 051504 (2012), arXiv:1208.4086 [hep-ph].

[28] P. Abreu et al. (Pierre Auger Collaboration), Phys.Rev.Lett. 109, 062002 (2012), arXiv:1208.1520 [hep-ex]

[29] G. Antchev et al. (TOTEM Collaboration), CERN-PH-EP-2012-354 (2012).

[30] D. Fagundes, M. Menon, and P. Silva, J.Phys. G40, 065005 (2013), arXiv:1208.3456 [hep-ph]

[31] J. Soffer, AIP Conf.Proc. 1523, 115 (2012), arXiv:1302.5045 [hep-ph],

[32] L. L. Jenkovszky, A. I. Lengyel, and D. I. Lontkovskyi, Int.J.Mod.Phys. A26, 4755 (2011), arXiv:1105.1202 [hep-ph], 